Irr

$$
\begin{aligned}
& \text { موسمية الإنتاج السمكي من المصايد الطبيعية في محافظة الفيوم }
\end{aligned}
$$

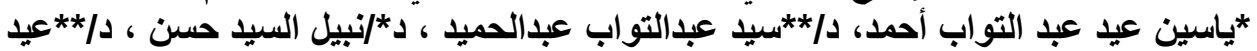

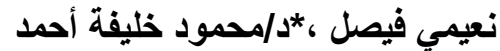

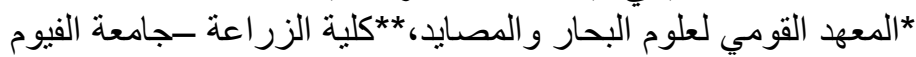

الملخص

يتميز الإنتاج السمكي من المصايد الطبيعية بمحافظة الفيوم المتمثلة في مصايد بحيرة

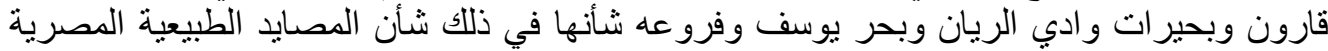

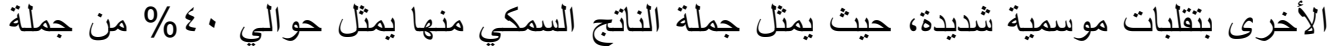

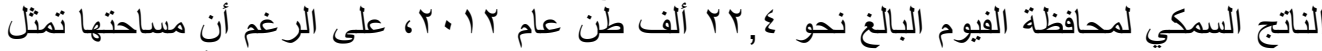

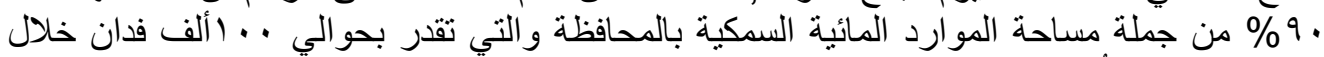

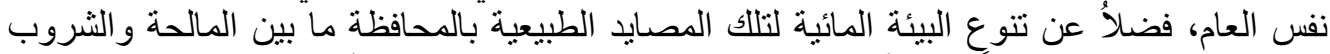

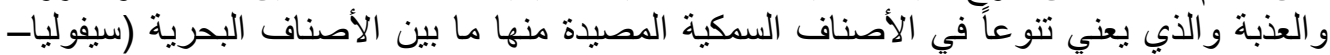

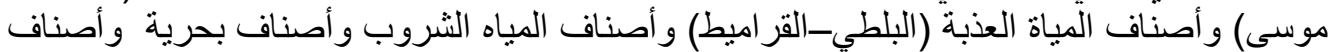

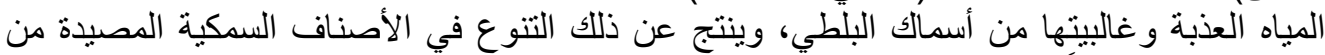

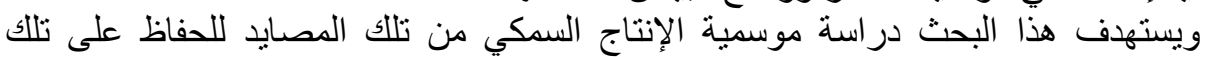

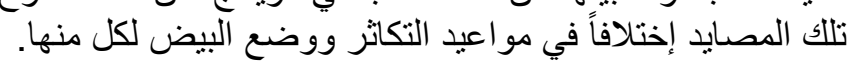

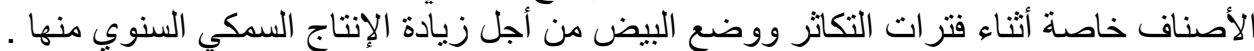

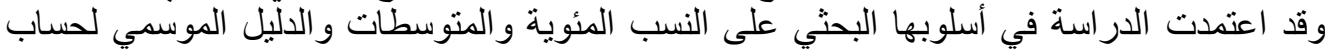

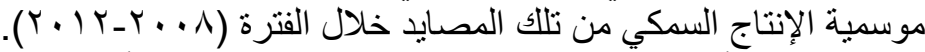

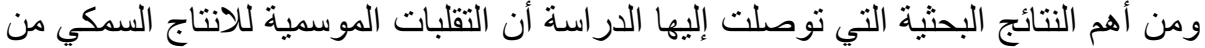

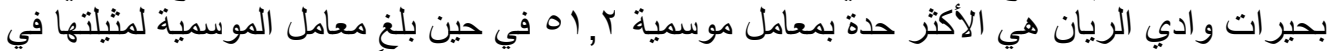

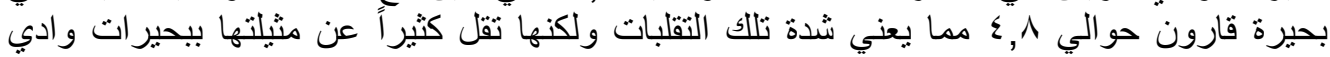

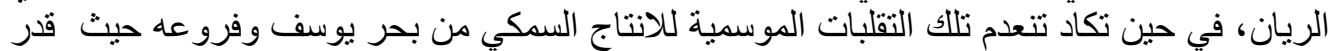

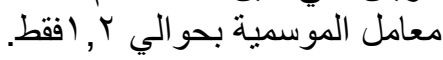

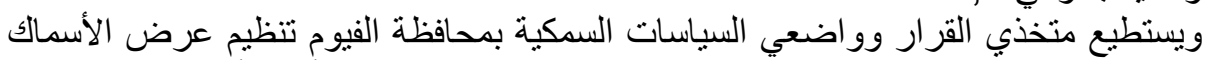

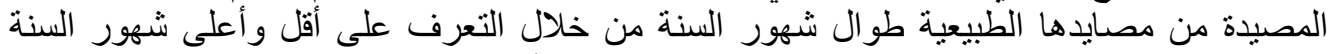

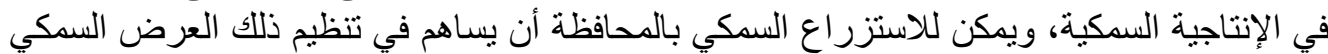

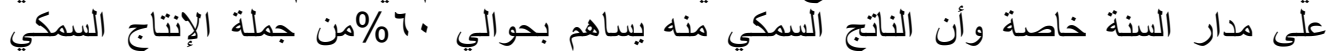

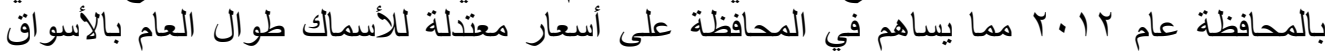

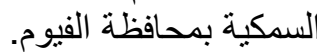

يتعرض الإنتاج السمكي كماً ونوعاً إلي تقلبات واسعة النطاق تسمى بالتقلقات التوات الموسمية

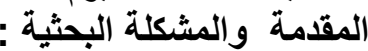

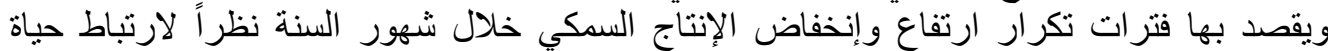

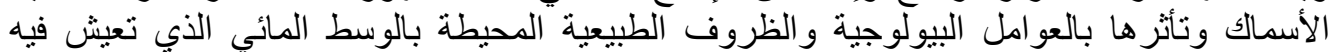

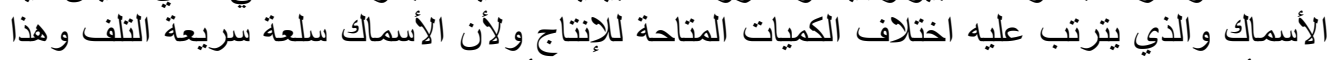

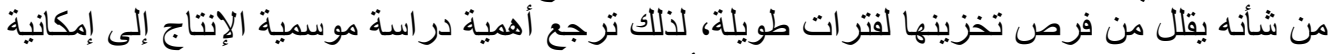

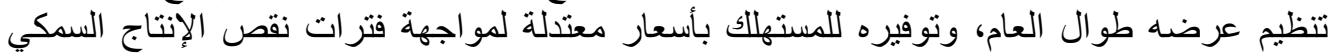

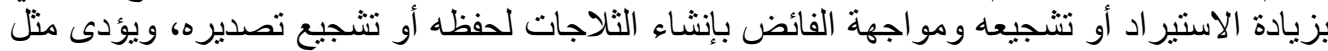

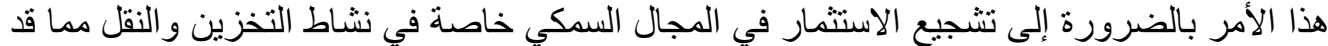

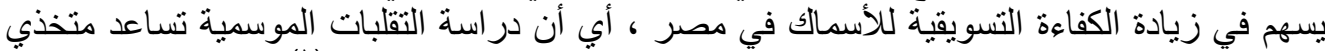

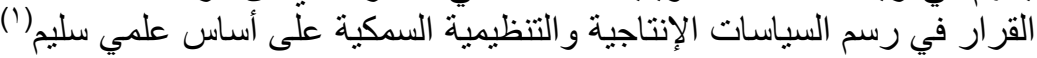

Fayoum J. Agric. Res. \& Dev., Vol. 30, No.1, July, 2016 
و المصايد الطبيعية بمحافظة الفيوم شأنها شأن المصايد الطبيعية المصرية يتميز الناتج

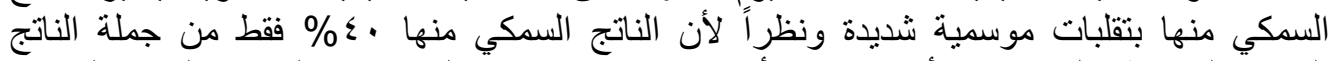

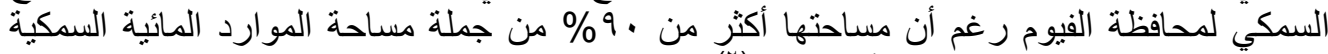

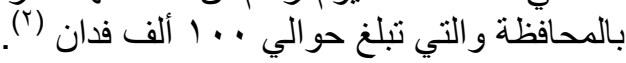

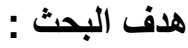

تستهدف الدراسة إبراز السمات الرئيسية لإنتاج الأسماك في المصايد الطبيعية في محافظة

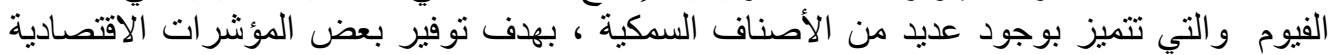

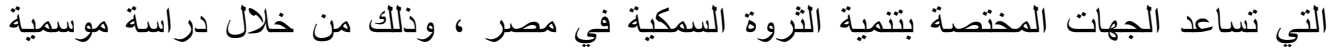
الإنتاج السمكي في المصايد الطبيعية في محافظة الفيوم .

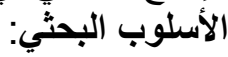

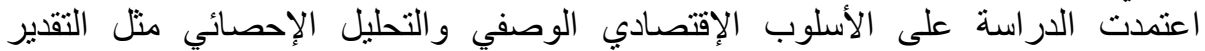

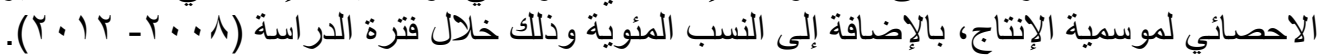

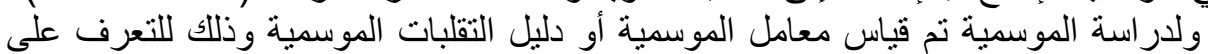

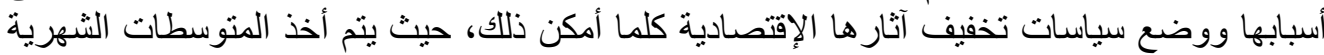

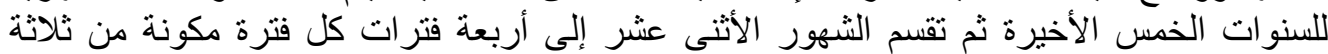

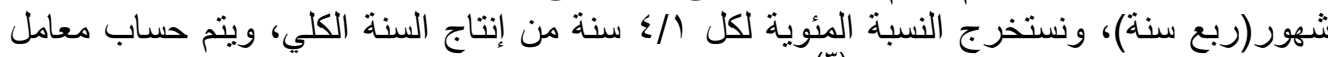

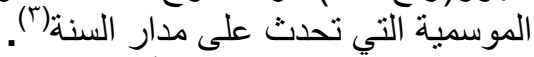

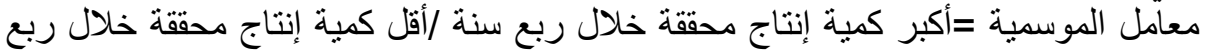

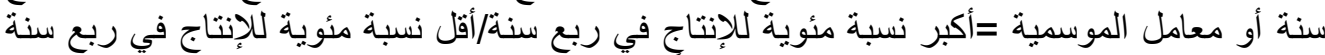

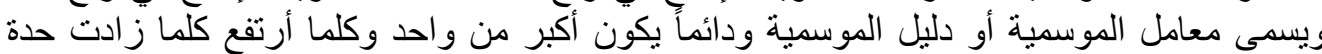

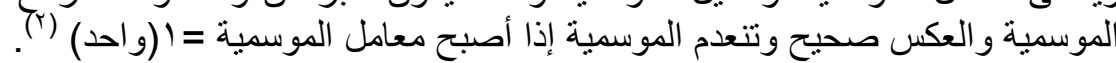

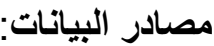
اعتمدت الدراسة على البيانات الإحصائية الثانوية المنشورة بمطبو عات الهيئة العامة لتنمية

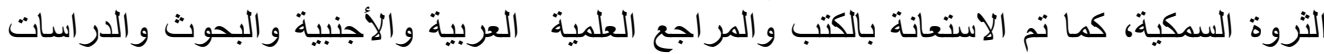

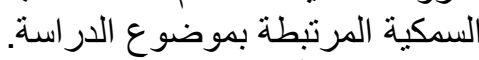
النتائج البحثية : البرتية

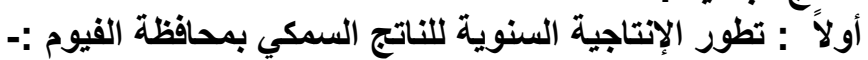

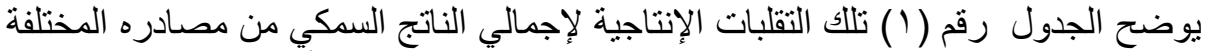

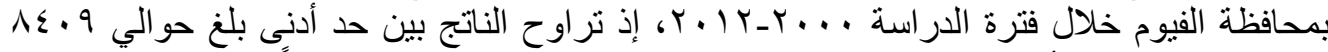

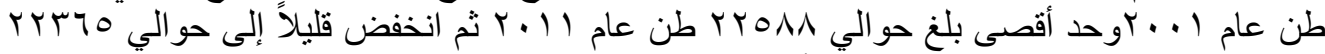

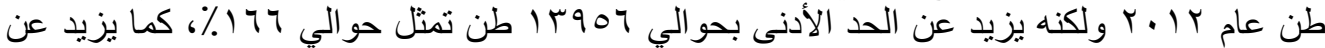

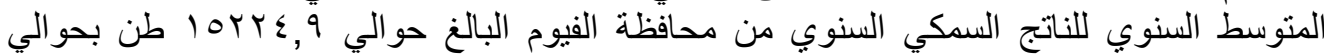

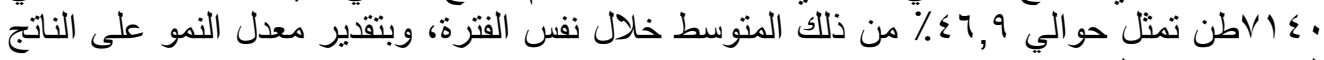
السمكي الإجمالي من مصنادره المختلفة من محافظة الفيوم تبين أن أفضل النماذج المقدرة هو النموذج النصف لوغاريتمي و ليتضح من النموذج السابق أن:

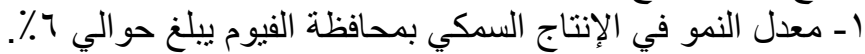

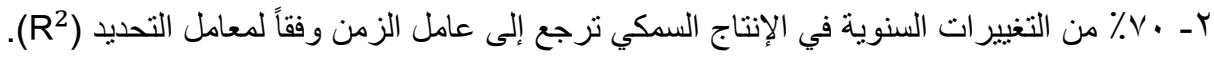

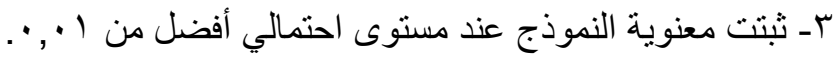
$\operatorname{Ln}=9856,848+0.057 \times$
$(11,096) * *(5,064) * *$
$\mathrm{R}^{2}=70$
$F=(25,640)$ 


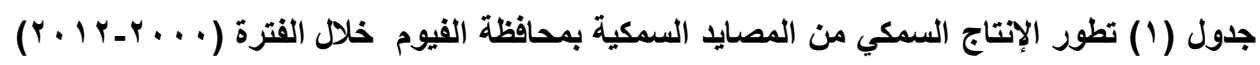

\begin{tabular}{|c|c|c|c|c|c|}
\hline الإجمالي بالطن & الاستزراع & نهر النيل & بحير الت واديان & بحيرة قارون & السنة \\
\hline 12360 & 8090 & 575 & 1876 & 1819 & 2000 \\
\hline 8409 & 5705 & 447 & 861 & 1396 & 2001 \\
\hline 11167 & 7601 & 450 & 1191 & 1925 & 2002 \\
\hline 14468 & 9187 & 1519 & 1310 & 2452 & 2003 \\
\hline 15547.6 & 8536,6 & 2703 & 1271 & 3037 & 2004 \\
\hline 15136.5 & 7070,5 & 3392 & 1992 & 2682 & 2005 \\
\hline 11916 & 4963 & 3614 & 1691 & 1648 & 2006 \\
\hline 15004 & 5655 & 4151 & 2126 & 3072 & 2007 \\
\hline 16324 & 8038 & 3047 & 2055 & 3184 & 2008 \\
\hline 16357 & 6913 & 3420 & 2624 & 3400 & 2009 \\
\hline 16282 & 8866 & 1019 & 2494 & 3903 & 2010 \\
\hline 22588 & 13530 & 1641 & 3053 & 4364 & 2011 \\
\hline 22365 & 13530 & 974 & 3451 & 4410 & 2012 \\
\hline lorrs, 9 & 8283,5 & 2073.2 & 1999,6 & 2868.6 & المتوسط \\
\hline 100 & 54,4 & 13,6 & 13,1 & 18,9 & $\%$ \\
\hline
\end{tabular}

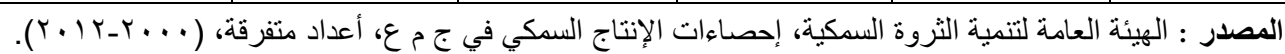

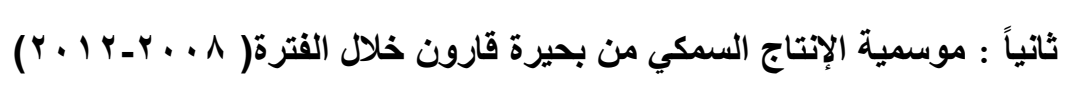

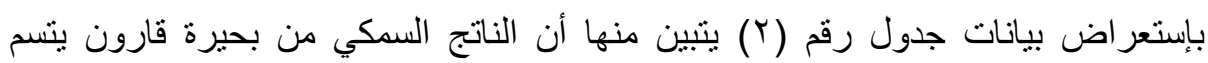

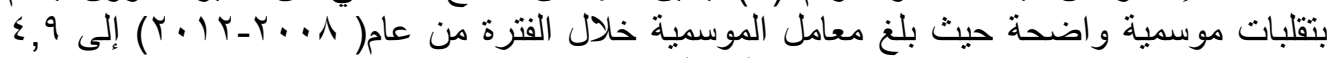

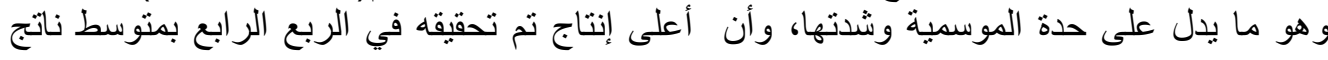

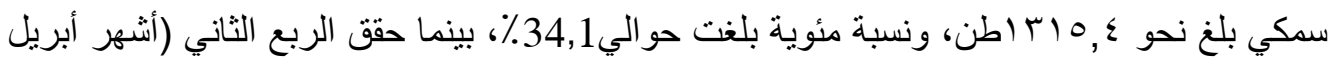

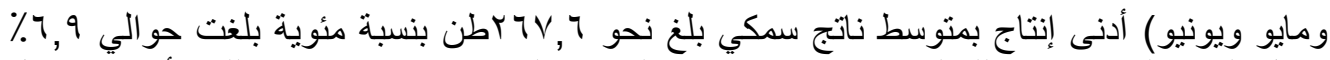

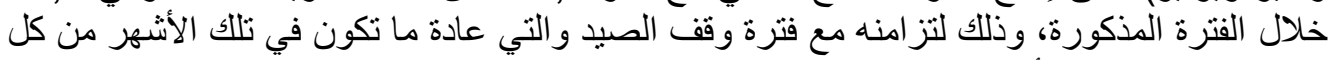
عام وهي فترة تكاثز أسماك البلطى.

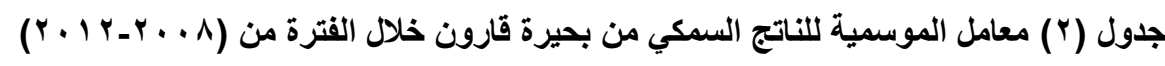

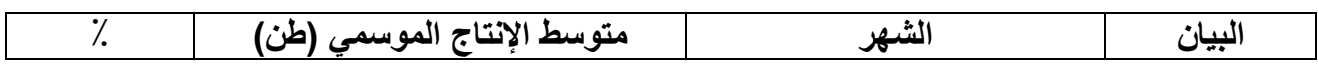

Fayoum J. Agric. Res. \& Dev., Vol. 30, No.1, July, 2016 
iro

\begin{tabular}{|c|c|c|c|}
\hline TV.r & $1.0 Y, r$ & يناير - فبر اير - مارس & الربع الأول \\
\hline 7,9 & YTV,T & أبريل- مايو - يونيو & الربع الثاني \\
\hline 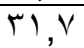 & $I Y I V, \varepsilon$ & يوليه- أغسطس- سبتمبر & الربع الثالثة \\
\hline$r \varepsilon .1$ & $1 \pi 10,2$ & أكتوبر - نو فمبر - ديسمبر & الربع الر ابع \\
\hline $1 \ldots$ & 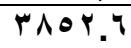 & - & الإجمالي \\
\hline
\end{tabular}

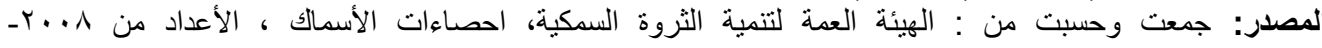

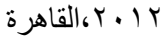

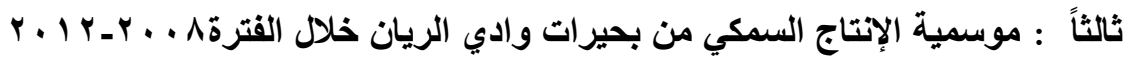

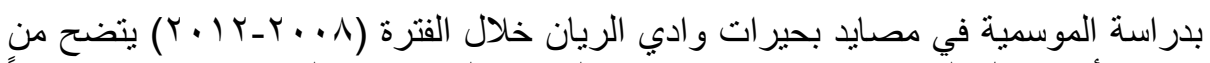

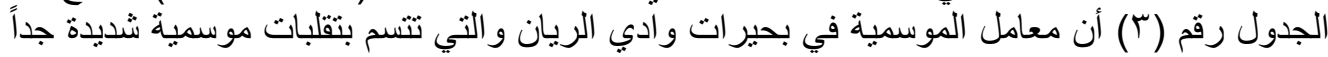

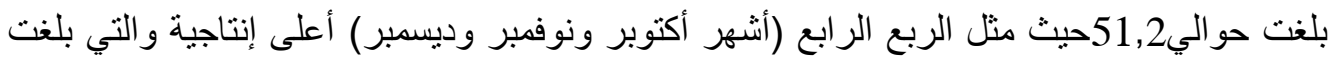

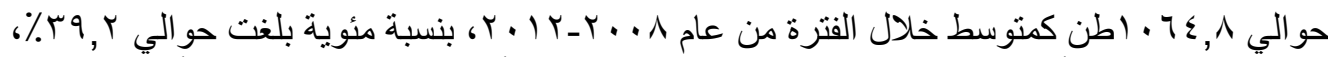
وقد يعود ذللك إلى أن هذا الربع يكون بداية موسم الصيد و وأو موسم ظهور وصيد أبيد أسماك العائلة

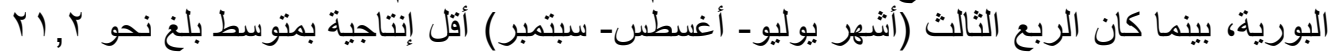

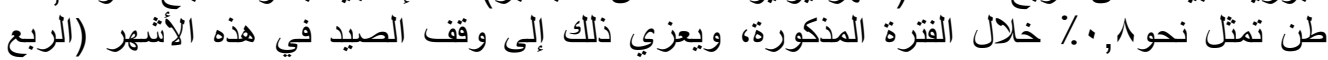

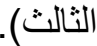

جدول () معامل الموسمية للناتج السمكي من بحيرات وادي الريان خلال الفترة من $(r+1 r-r+. \Lambda)$

\begin{tabular}{|c|c|c|c|}
\hline$\%$ & متوسط الإنتاج الموسمي(طن) & الشهر & البيان \\
\hline ro.^ & $9 \Lambda \cdot, r$ & يناير - فبر اير - مارس & الربع الأول \\
\hline KT.V & $7 \leqslant \wedge, \wedge$ & أبريل- مايو - يونيو & الربع الثاني \\
\hline.$\wedge$ & $r 1, r$ & بوليه_أغسطس- سبتمبر & الربع الثالث \\
\hline rq.V & $1 \cdot \lambda \leq . \wedge$ & أكتوبر -نو فمبر - ديسمبر & الربع الرابع \\
\hline $1 \cdots$ & TV10 & 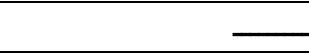 & الإجمالي \\
\hline
\end{tabular}

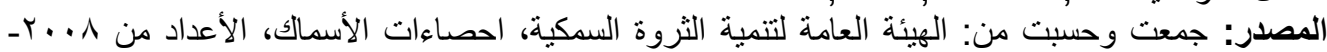

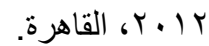

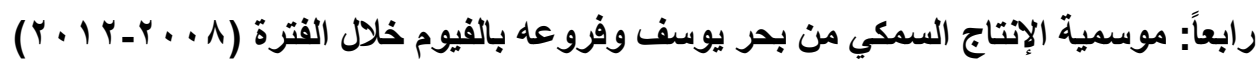

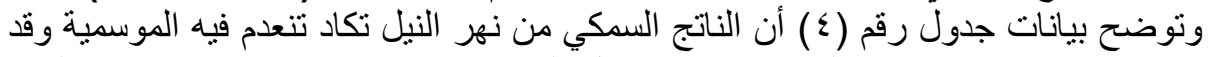

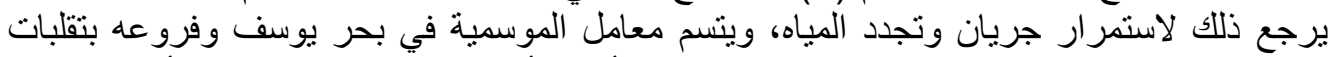

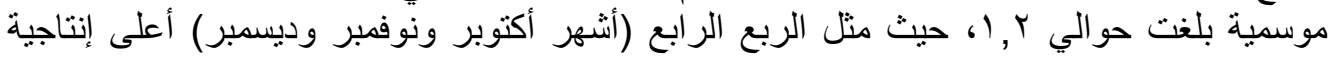

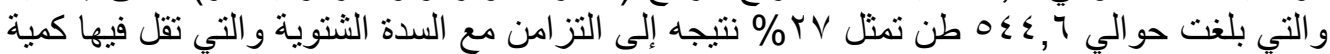

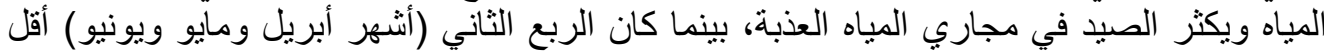

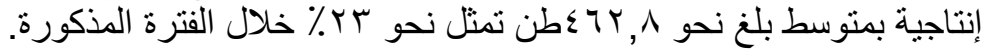

جلول (؛) معامل الموسمية للناتج السمكي من نهر النيل (بحر يوسف وفروعل) خلال الفترة من(

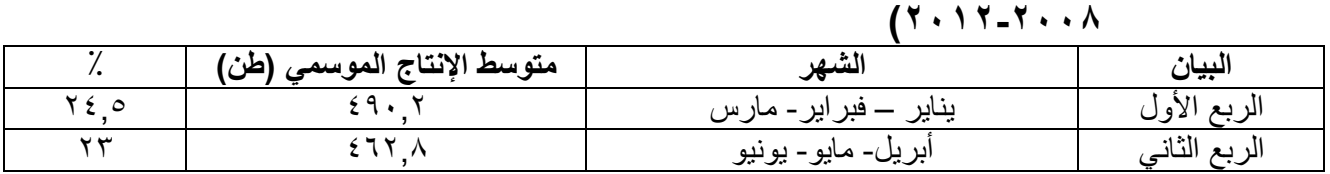

Fayoum J. Agric. Res. \& Dev., Vol. 30, No.1, July, 2016 


\section{Y Y}

\begin{tabular}{|c|c|c|c|}
\hline ro,o & $01 Y, 7$ & يوليه - أغسطس- سبتمبر & الربع الثالث \\
\hline tr & $0 \leqslant \leqslant, 7$ & أكتوبر - نوفمبر - ديسمبر & الربع الرابع \\
\hline $1 \ldots$ & $r \cdot 1 \cdot, r$ & 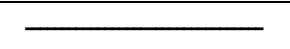 & الإجمالي \\
\hline
\end{tabular}

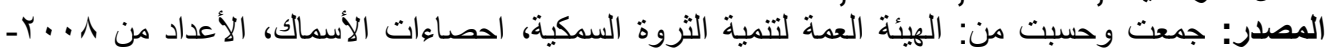

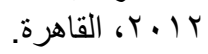

$$
\text { وتوصي الاراسة: }
$$

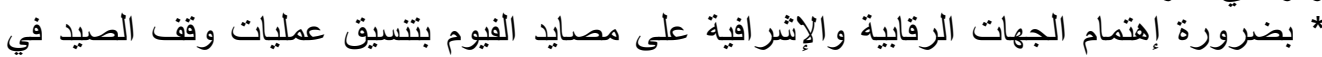

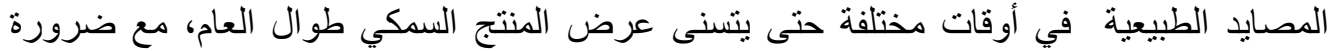

$$
\text { تنظيم العرض في الاستزر اعلئ السمكي. }
$$

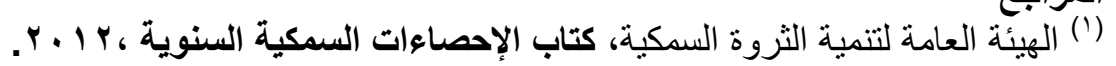

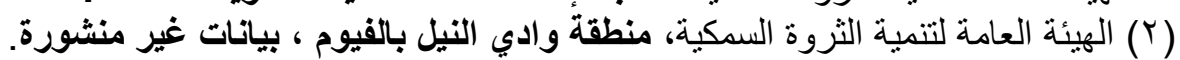

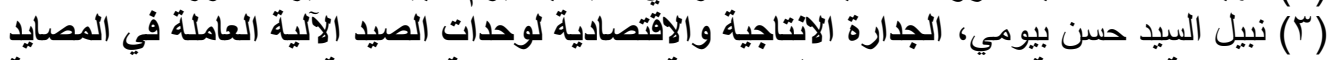

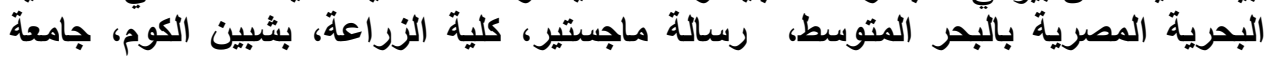

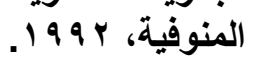

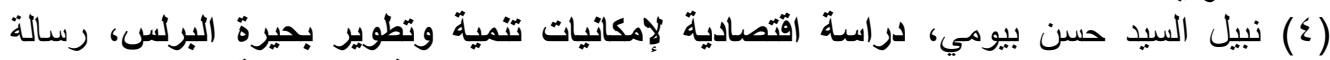

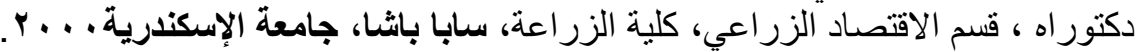

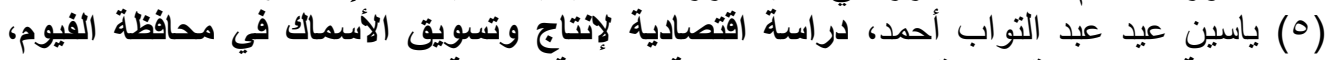

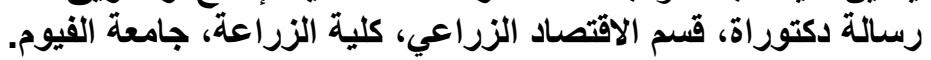

SEASONALITY OF FISH PRODUCTION OF NATURAL FISHERIES IN EL-FAYOUM GOVERNORATE

Abd El-Tawab Y. Eid, Abd EL-Hameed S. Abd El-Tawab , Hassan N. El-sayed , Hassan E. Naeemy, Ahmad M . Khaleefaa

Fayoum J. Agric. Res. \& Dev., Vol. 30, No.1, July, 2016 
Fish production from natural fisheries in El-Fayoum Governorate at is characterized by a fisheries of Qarun lake, Wadi El-Rayan lakes and Bahr Youssef and its canals, like other Egyptian natural fisheries seasonal fluctuations severe.

Science the enter product of fish catch which represents about $40 \%$ of total fish catch from El-Fayoum Governorate fisheries which phrase about 22.4 thousand tons in 2012 despite the fact that water area represents more than $90 \%$ of the total fish water resources preservation area estimated at about 100 thousand feddan during the same year, as well as the diversity of environment and water to those of natural fisheries to maintain between saline , brackish and fresh which means diverse fish species trap them between marine species such as Solia and Mullet, and varieties of fresh water such as Tilapia and catfish varieties brackish water mostly fresh water fish, followed by the diversity of the trap varieties of those fisheries the different in breeding seasons dates and lay eggs each.

This research is aims to study seasonality of fish production from those fisheries to keep those items specially during periods of breeding and laying eggs in order to increase the annual fish catch from them The study based on the use of averages, percentages and seasonal guide to calculate the seasonality of fish production from these natural fisheries during the period 2008-2012.

Among the most important research findings of the study that the seasonal fluctuations of fish production from Wadi El-Rayan lakes is the most sever and the inter intensity by a factor of seasonal estimated at about 51.2, while this factor in karon lake at about 4.8 which means high severity but far less than from those Wadi El-Rayan lakes And virtually no such seasonal fluctuations of fish productions Bahr Youssef and its canals by a factor of as much as about 1.2 only.

Decision - makers in El-Fayoum governorate can organize the Fish supply which caught from the natural fishery throughout the months of the year by identifying the lowest and the highest fish production months. Fish culture can preservation contribute to the organization that fish supply throughout the private and the resulting fish than a year it contributes about $60 \%$ of total fish catch in El-Fayoum Governorate in 2012 , which would maintain the moderate prices of fish in fish markets throughout the year in ElFayoum Governorate.

Fayoum J. Agric. Res. \& Dev., Vol. 30, No.1, July, 2016 